\title{
Interessengemeinschaft Disease Management IGDM
}

\author{
P. Trautmann, namens der Interessengemeinschaft Disease Management IGDM
}

Die IGDM ist eine Initiative grosser Krankenversicherer zur Förderung von Disease Management in der Schweiz. Mit dem folgenden Artikel soll eine Diskussion über den Stellenwert von Disease Management im Schweizer Gesundheitswesen lanciert werden. Besonders angesprochen sind kantonale Ärztegesellschaften und medizinische Fachgesellschaften.

\section{Was ist Disease Management?}

In der Literatur findet sich keine allgemein akzeptierte Definition von Disease Management. Disease Management verfolgt letztlich die effektivitäts- und effizienzsteigernde Bündelung und Koordination bisheriger Konzepte zur Abklärung und Behandlung chronischer Krankheiten unter aktivem Einbezug der Patienten. Gemäss Definition der Disease Management Association of America (DMAA) umfassen Disease-Management-Programme sechs Komponenten: Prozesse zur Identifikation der Patienten, evidenzbasierte Abklärungs- und Behandlungsrichtlinien, koordinierte Zusammenarbeit zwischen allen Therapeuten, Schulung der Patienten im Self-Management, Outcome-Analysen und klare Kommunikationswege zwischen allen Beteiligten.

\section{Warum ist Disease Management gerade jetzt ein Thema?}

Disease-Management-Programme werden heute von fast allen wichtigen Gruppen im Schweizer Gesundheitswesen als Instrumente anerkannt, welche dazu beitragen, die Effektivität und Effizienz der medizinischen Versorgung chronisch kranker Menschen zu verbessern. Für die Patienten und ihre Angehörigen steht die durch Disease-Management-Programme verbesserte Lebensqualität natürlich an erster Stelle.

Wir stellen aber fest, dass sich solche Programme in der Schweiz noch nicht im erwarteten Mass etabliert haben. Die mit Disease Management beschäftigten Akteure verfügen über sehr gute Kenntnisse, die wesentlichen Bestandteile der benötigten Infrastruktur sind vorhanden, und mehrere Pilotprojekte sind initiiert und durchgeführt worden. Trotzdem ist es bis heute nicht gelungen, insbesondere die Leistungser- bringer vom Nutzen von Disease-ManagementProgrammen zu überzeugen. Entsprechend klein blieb die Zahl von Patienten, die bisher in Disease-Management-Programmen betreut wurden. Auch die Krankenversicherer haben DiseaseManagement-Programme bis jetzt nur verhalten unterstützt, nicht zuletzt auch aus Angst vor einer reverse selection.

Durch die zunehmende Prävalenz chronischer Krankheiten und die damit einhergehende ungebremste Kostenentwicklung steigt die Attraktivität von Disease-Management-Programmen kontinuierlich.

\section{Was will die Interessengemeinschaft Disease Management IGDM?}

Um die Erfolgschancen von Disease-Management-Programmen in Zukunft zu erhöhen, bietet sich die Kooperation interessierter Krankenversicherer an. Damit können zwei Hauptziele erreicht werden: einerseits ermöglichen die höheren Patientenzahlen eine aussagekräftige Outcome-Analyse als Voraussetzung für zunehmende Akzeptanz von Disease Management auch in der Schweiz, und andererseits wird das Risiko der «reverse selection» durch die Teilnahme mehrerer grosser Krankenversicherer nahezu eliminiert. Mit diesen wissenschaftlich erhobenen Daten möchte die IGDM insbesondere auch die Diskussion über den zukünftigen Stellenwert von Disease Management im Schweizer Gesundheitssystem lancieren.

Die Krankenversicherer Helsana, Groupe Mutuel, Wincare, Visana und CSS und die SanaCare AG sind aktuell in der IGDM zusammengeschlossen. Sie fördern gemeinsam die Entwicklung, Etablierung und den Betrieb von Disease-Management-Programmen im Behandlungsprozess chronischkranker Patienten. Hauptsächlicher Beweggrund der Beteiligten ist die Erkenntnis, dass ein Alleingang in Sachen Disease Management aus den oben erwähnten Gründen wenig erfolgversprechend ist. Die IGDM versteht sich vorläufig als informelle Arbeitsgruppe, die bisher ohne eigene professionelle Strukturen auskommt. 
Warum ist die Indikation Herzinsuffizienz das erste gemeinsame DiseaseManagement-Programm der IGDM?

Die in der IGDM vertretenen Krankenversicherer werden im Jahr 2005 als erstes gemeinsames Disease-Management-Programm das bereits seit einiger Zeit bestehende Herzprogramm lancieren. Mit dem koordinierten Vorgehen der IGDM sollen innert kurzer Zeit genügend neue Patientinnen und Patienten in diesem Disease-Management-Programm betreut werden, um statistisch signifikante Outcome-Daten erheben zu können.

Das Herzprogramm ist ein spezifisches Schulungs- und Betreuungsprogramm für Patienten mit Herzinsuffizienz. Die Verantwortung für die medizinische Behandlung bleibt weiterhin bei den von den Patienten gewählten Ärzten, das heisst beim bisherigen Hausarzt oder Kardiologen. Ein Arztwechsel wird ausdrücklich nicht beabsichtigt, auch werden vom Herzprogramm keine Anpassungen der medikamentösen Therapie vorgenommen. Die Behandlung wird aber unterstützt, indem die Patienten selbst eine aktivere Rolle einnehmen.

In vergleichbaren Programmen konnten mit spezifischen Betreuungs- und Interventionsmassnahmen die Notfallhospitalisationen deutlich gesenkt und eine Reduktion von Morbidität und Behandlungskosten erreicht werden. Weiter zeigen die bisher vorliegenden Erfahrungen, dass die Konsultationsrate beim behandelnden Arzt im Rahmen solcher Programme leicht zunimmt, da sich die Patienten etwas häufiger an ihn wenden. Dafür sinken die Hospitalisationsraten und die Gesamtkosten signifikant [1-3].

Die Kosten des Programms werden von den aktuell in der Interessengemeinschaft Disease Management vertretenen Krankenversicherern CSS, Helsana, Groupe Mutuel, Visana und Wincare übernommen.

Interessierten Kreisen, insbesondere Kantonal- und Fachgesellschaften, stehen wir gerne für einen weiterführenden Austausch zur Verfügung. Wir freuen uns auf Ihre Kontaktaufnahme.

Literatur

1 Stewart S, Markley JE, Horowitz JD. Effects of a multidisciplinary, home-based intervention on planned readmissions and survival among patients with chronic congestive heart failure: a randomised controlled study. Lancet 1999; 354:1077-83.

2 McAlister FA, Lawson FM, Teo KK, Armstrong PW. A systematic review of randomised trials of disease management programs in heart failure. Am J Med 2001;110:378-84.

3 Meyer K, Suter TM. Die chronische Herzinsuffizienz erfordert ein multidisziplinäres Behandlungskonzept. Praxis 2001;90:1766-74. 